\title{
LOCAL PROPAGATOR THEORY
}

\author{
STANLEY STEINBERG
}

\begin{abstract}
A survey of abstract propagator theory (Ovcyannikov or Ovsjannikov Theorem) is given. This theory is reminiscent of semigroup theory. However, while semigroup theory is appropriate to the study of well posed initial value problems, abstract propagator theory is most appropriate to the study of (so called) ill posed initial value problems. A list of elementary ill posed problems is presented and references to deeper and more difficult problems are given.

Next, an exposition of the basic abstract structure and elementary linear theory along with some elementary examples in given. This is followed by a survey of the most recent linear theory, nonlinear theory, and applications.
\end{abstract}

\section{CONTENTS}

I. Elementary Theory

0 . Introduction

1. The abstract set up and examples

2. A linear theorem

3. A linear Cauchy-Kowalewski theorem

4. Functorial remark

5. Holmgren's uniquess theorem

6. More scales of Banach spaces

II. General Theory

0 . Introduction

1. The linear theorem

2. Example

3. Nonlinear theorems

4. Lie algebras and scales

I. Elementary Theory. 0. Introduction. In this paper we shall describe an abstract theory which is useful in studying various initial value problems for partial differential equations. The theory involves the solvability of an abstract initial value problem in a continuous family (usually called a scale) or sequence of Banach spaces. The results are usually referred to as the Ovcyannikov (or Ovsjannikov) theory or as an Abstract Cauchy-

Received by the editors on November 15, 1976 and in revised from on March 3, 1979. 
Kowalewski theorem. We prefer to call these results "abstract propagator theory", where by a propagator we mean an operator that maps the initial data of an initial value problem into a solution of the problem at some positive time.

The first appearance of a version of this theorem that used the concept of a scale or a sequence of Banach spaces is in Gel'fand and Shilov [12], v. 3 page 94, in 1958 and later, but independently, by T. Yamanaka [46], 1960, L.V. Ovcyannikov [26], 1965 and F. Treves [43], 1968. There are currently many versions of these results including those in Ovcyannikov [25], Du Chateau [8], Nirenberg [22], Yamagata [45], Steinberg [34] and Baouendi and Goulaouic [3]. The cited literature shows that this abstract theory has many deep and important consequences in the theory of the initial value problem in partial differential equations. In order to give the reader an idea of the types of applications, we give the following list of elementary examples. The cited literature gives applications to much more general problems.

A. Cauchy-Kowalewski and Holmgren's Theorems. We note that these techniques can be used to obtain the most general nonlinear form of the Cauchy-Kowalewski Theorem, that is, where the data is analytic in the space variables and only continuous in the time variable; see Nirenberg [22]. It appears that any existence and uniqueness results for initial value problems that can be obtained using power series techniques can be obtained more easily using abstract propagator techniques. Also the dual theory can be used to obtain Holmgren's uniqueness theorem (see $[33,7,31])$.

B. The parametric osillator equation. The parametric oscillator equation is given by

$$
\begin{gathered}
\frac{\partial P}{\partial t}=-\frac{1}{2}\left(\frac{\partial^{2} P}{\partial x^{2}}-\frac{\partial^{2} P}{\partial y^{2}}\right)+x \frac{\partial P}{\partial x}-y \frac{\partial P}{y \partial}, P=P(x, y, t) \\
P(x, y, 0)=P_{0}(x, y)
\end{gathered}
$$

for $(x, y, t) \in \mathbf{R}^{2} \times[0, T]$. This equation belongs to a class of equations

$$
\partial P / \partial t=Q(x, y, \partial / \partial x, \partial / \partial y) P
$$

where $Q$ is a quadratic expression in $x, y, \partial / \partial x, \partial / \partial y$ and where no symmetry assumptions are placed on $Q$. Note that the right hand side of the parametric oscillator equations has principal part which is the wave equation and consequently the parametric oscillator equation in not of classical type. For an extensive discussion of these equations, see Steinberg and Treves [38], where there are also references to many discussions of equations of this type in the physics literature. However, we will make 
a few comments on the solutions of this problem. First, the solutions bear some resemblance to the solutions of the backwards heat equation (explicit solutions of the parametric oscillator equation can be found). For the initial data $\exp \left(-\alpha\left(x^{2}+y^{2}\right)\right)$, there exists a classical solution of the parametric oscillator equation for a finite interval of time. If one allows the solution to be an analytic functional, then there exists a solution global in time. This may at first seem inconvenient, but the quantities of physical interest are the moments of the solution (not the values at a point) and analytic functionals possess well behaved moments, so this type of solvability is appropriate to this situation.

C. Shot-put noise. The equations that appear in shot-put noise are of infinite order; for instance,

$$
\begin{gathered}
\partial f / \partial t=F(t, \partial / \partial x) f, \quad f=f(x, t) \\
f(x, 0)=f_{0}(x) \\
F(t, \xi)=\sum_{n=0}^{\infty} a_{n}(t) \xi^{n} .
\end{gathered}
$$

In some elementary cases some insight can be gained into the solvability of these equations by the use of the Fourier transform. For a more extensive treatment see Steinberg [37]. In some applications the equations are truncated to second order and it is still unknown under what conditions this approximation is valid.

D. The Cauchy-Goursat problems. An elementary problem of this type is

$$
\begin{gathered}
\frac{\partial^{2} f}{\partial t_{1} \partial t_{2}}=P\left(x, t_{1}, t_{2}, \frac{\partial}{\partial x}\right) f, \quad f=f\left(t_{1}, t_{2}, x\right) \\
f\left(0, t_{2}, x\right)=f_{1}\left(t_{2}, x\right), \quad t_{2} \geqq 0 \\
f\left(r_{1}, 0, x\right)=f_{2}\left(t_{1}, x\right), \quad t_{1} \geqq 0
\end{gathered}
$$

where $P$ is some partial differential operator. For an extensive discussion of this type of problem see DuChateau [8].

E. Degenerate equations or equations with regular singular points. A simple problem of this type is

$$
\begin{gathered}
t \frac{\partial f}{\partial t}+A\left(x, t, \frac{\partial}{\partial x}\right) f=g, \quad f=f(x, t), \quad g=g(x, t) \\
f(x, 0)=?
\end{gathered}
$$

where $A$ is a regular partial differential operator and $g$ is a given function. For a discussion of higher order cases and a discussion of what initial data is to be specified see Baouendi and Gaoulaouic $[1,2]$. 
F. Hydrodynamics. Let us consider the following simple problem.

$$
\begin{gathered}
\frac{\partial f}{\partial t}=a \frac{\partial f}{\partial \theta}+b \frac{\partial f}{\partial r}+c f, \quad f=f(\theta, t) \\
f(\theta, 0)=f_{0}(\theta)
\end{gathered}
$$

where $f(\theta, t)$ and $f_{0}(\theta)$ are to be periodic of period $2 \pi$ in $\theta$ and $a, b, c$ are real constants. Here $\partial / \partial r$ is a shorthand notation for the following operator. Let $u(r, \theta, t)$ be harmonic in the unit disc with $u(1, \theta, t)=f(\theta, t)$ and then set

$$
\frac{\partial f(\theta, t)}{\partial r}=\left.\frac{\partial u(r, \theta, t)}{\partial r}\right|_{r=1}
$$

Using the Poisson kernel one can find an explicit expression for $\partial f / \partial r$ and then one can easily verify that this operator is a non-local pseudodifferential operator of order 1 . It is also possible to solve this problem explicitly, using Fourier series and see that the problem is not classically well posed, that is, the equation has a non-real characteristic.

This equation is a simple prototype for the equations describing the movement of a bubble in a fluid. The equation corresponds to the boundary condition on the surface of the bubble in Lagrangian coordinates. For further discussions of these problems see Ovcyannikov $[25,26]$. We note that there are many problems in hydrodynamics to which these techniques should apply.

G. Hyperbolic equations with multiple characteristics. A simple example of this type of equation is

$$
\begin{array}{rlrl}
\frac{\partial^{2} f}{\partial t^{2}} & =\frac{\partial f}{\partial x}, & & f=f(x, t) \\
f(x, 0) & =f_{0}(x), & \frac{\partial f}{\partial t}(x, 0)=f_{1}(x) .
\end{array}
$$

Note that this problem corresponds to a physical problem where one measures the temperature and flux at one point for all time and then attempts to find the heat distribution. Also note that the principal part of our operator is $\partial^{2} / \partial t^{2}$ which is hyperbolic with respect to $t=0$ and has a double characteristic and also that the problem is easily solved using the Fourier transform. Using this explicit formula it is easily seen that the problem is not classically well posed. Our techniques can be used to study the initial value problem for higher order equations or systems of equations that are linear but may have multiple characteristics. The main hypothesis on the equations studied is that the characteristics be real and smooth functions of the space and time variables. We also 
note that solvability is obtained in spaces of Gevrey functions, or their duals, which can be seen to be a natural choice by looking at examples similar to the one above.

Equations of this type appear in magnetohydrodynamics [6]. For a discussion of these problems see Steinberg [34]. It should be possible to extend these techniques to nonlinear problems. Also we note that there is a relationship between the techniques in [38] and those of Beals [5] and Leray and Ohya [18] for studying problems with multiple characteristics.

We note that in all the previous examples the abstract propagator technique produces solvability results in spaces of functions which are subspaces of the infinitely differentiable functions, such as analytic functions, entire functions, or Gevrey functions, or in the duals of such spaces. We wish to contrast this with the semi-group, energy or other methods used to study strictly hyperbolic, parabolic or Schroedinger type equations and which give solvability in spaces of functions which are not subspaces of the infinitely differentiable functions. Also, the abstract propagator technique always gives existence, uniqueness and continuous dependence on the parameters of the problem. The continuous dependence is given in either very strong or very weak topologies. In general, the dependence on the time variable need only be continuous or integrable. We can obtain both local and global existence in the time variable; however, the technique is basically local in the time variable.

In general we use the notation of modern P.D.E. theory [15]. References $[1-4,7-10,12,22,23,25,26,28,30-38,41-43,45-47]$ are to papers that use local propagator theory directly, while references $[5,6,11,13,16-18,20,24,27,29,39,40]$ are to papers that do not use local propagators but whose results have been obtained (or should be able to be obtained) by using these techniques, and references $[14,15,19,21,44]$ are in neither of the above categories. This paper is broken into two parts; the first gives an elementary version of the theory while the second gives the general results.

These notes were developed in a seminar given in the Department of Mathematics and Statistics, University of New Mexico. I wish to thank the participants and, in particular, Professor Reuben Hersh for many helpful suggestions. I would also like to thank the referee for several helpful suggestions.

1. The abstract set up. We begin by setting up an abstract structure.

Definition 1.1. Let $I$ be an open interval of real numbers. For each $s \in I$, let $X_{s}$ be a Banach space with norm $\|\cdot\|_{s}$. If for all $s, \sigma \in I$ we have

i) $X_{s} \leqq X_{\sigma}$ for $\sigma \leqq s$, and

ii) $\|x\|_{\sigma} \leqq\|x\|_{s}$ for $x \in X_{s}$, 
then $S=\left\{X_{s}, I\right\}$ is called a scale of Banach spaces on $I$.

Definition 1.2. We will say that $A$ is a bounded operator on $S$ if for every $s, \sigma \in I, \sigma<s, A$ is a bounded mapping of $X_{s}$ into $X_{\sigma}$, i.e., $A \in$ $L\left(X_{s}, X_{\sigma}\right)$.

Remark. $L\left(X_{s}, X_{\sigma}\right)$ is given the usual norm topology, that is, the uniform operator topology.

Definition 1.3. If $0<d<\infty$, and $A$ is a bounded operator on the scale $S$, then $A$ is of type $d$ on $S$ if there is a constant $C$ such that

$$
\|A x\|_{\sigma} \leqq C(s-\sigma)^{-d}\|X\|_{s}
$$

for all $s, \sigma \in I, \sigma<s$ and all $x \in X_{s}$.

Remark. The anove can be stated somewhat more precisely by supposing there are injection maps

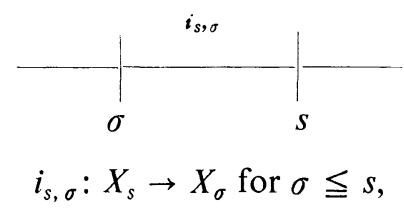

that is, $i$ is $a 1-1$ linear map with dense range and

$$
i_{s, \sigma} \circ i_{\sigma, s^{\prime}}=i_{s, s^{\prime}} \text { for } s^{\prime} \leqq \sigma \leqq s .
$$

Also, the mapping

$$
\begin{gathered}
A=A_{s, \sigma}: X_{s} \rightarrow X_{\sigma} \text { for } \sigma \leqq s \\
A_{s, \sigma} \circ i_{\sigma, s^{\prime}}=A_{s, s^{\prime}}
\end{gathered}
$$

depends on $s$ and $\sigma$. We will usually suppress the $i_{s, \sigma}$ map and the dependence of $A$ on $s$ and $\sigma$ because no confusion is likely to result.

ExAmple. Let $I=(0, \infty)$ and $X_{s}=\{f: f$ is analytic for $|z|<s, f$ is bounded and continuous for $|z| \leqq s\}$,

$$
\|f\|_{s}=\sup _{|z| \leq s}|f(z)|=\sup _{|z|=s}|f(z)| .
$$

If $f \in X_{s}$, then $f \in X_{\sigma}$ for $\sigma<s$ and

$$
\sup _{|z| \leqq \sigma}|f(z)| \leqq \sup _{|z| \leqq s}|f(z)| \text { or }\|f\|_{\sigma} \leqq\|f\|_{s} .
$$

Also note that the polynomials are dense in $X_{s}$, so $X_{s}$ is dense in $X_{\sigma}$. Finally

$$
\begin{aligned}
\left\|\frac{d f}{d z}\right\|_{\sigma} & \leqq(s-\sigma)^{-1}\|f\|_{s} \\
\|f g\|_{s} & \leqq\|f\|_{s}\|g\|_{s} .
\end{aligned}
$$


The first inequality is called the Schwarz-Pick lemma and the second is trivial. We will return to this example.

Proposition 1.1. If for $i=1,2, A_{i}$ is of type $d_{i}$ in $X_{s}$, then $A_{1} \circ A_{2}$ is of type $d_{1}+d_{2}$.

Proposition 1.2. If $f$ is a continuously differentiable mapping of the interval $J$ into the interval $I$ with $f^{\prime} \geqq 0$ and $X_{s}$ is a scale on $I$, then $S^{\prime}=$ $\left\{Y_{s}, J\right\}, Y_{s}=X_{f(s)}$, is a scale on $J$. Moreover, if there exists a constant $M>0$ such that $f^{\prime} \geqq M$ and $A$ is of type $d$ on $S$, then $A$ is of type d on $S^{\prime}$.

The proofs of Propositions 1.1 and 1.2 are left to the reader.

ExAmple. Let us now study the following simple situation. Let $A$ be of type $d$ on $S=\left\{X_{s}, I\right\}$. We wish to define the operator

$$
e^{A t}
$$

operating on $X_{s}$. First note that $A$ being of type $d$ implies that

$$
\|A x\|_{\sigma} \leqq C(s-\sigma)^{-d}\|x\|_{s}, x \in X_{s} .
$$

We now show by induction that

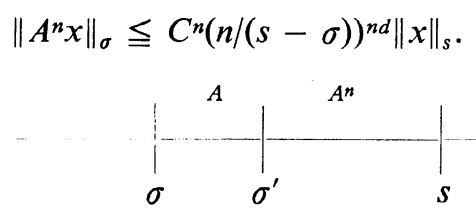

If we choose $\sigma^{\prime}$ such that $\sigma<\sigma^{\prime}<s$, then

$$
\left\|A^{n+1} x\right\|_{\sigma} \leqq C\left(\sigma^{\prime}-\sigma\right)^{-d} C^{n}(n /(s-\sigma))^{n d}\|x\|_{s} .
$$

Choose $\sigma^{\prime}-\sigma=\varepsilon(s-\sigma)$ and $\varepsilon=1 /(n+1)$ so that

$$
\begin{aligned}
\left\|A^{n+1} x\right\|_{\sigma} & \leqq C^{n+1}\left[\varepsilon^{-1}(1-\varepsilon)^{-n}\right]^{d}(s-\sigma)^{-(n+1) d} \\
& \leqq C^{n+1}(n+1)^{(n+1) d}(s-\sigma)^{-(n+1) d} .
\end{aligned}
$$

Next, let $a \in I$ and $x \in X_{a}$ and define

$$
e^{A t} x=\sum_{n=0}^{\infty} \frac{t^{n} A^{n}}{n !} x .
$$

Now this series is dominated in $X_{s}$ term-by-term by the series

$$
\sum_{n=0}^{\infty} \frac{t^{n}}{n !} C^{n}(n /(a-s))^{n d}\|x\|_{s}
$$

for $s<a$, that is, 


$$
\sum_{n=0}^{\infty}\left(\frac{t C}{(a-s)^{d}}\right)^{n} \frac{n^{n d}}{n !} .
$$

Note that

$$
n^{n} / n ! \leqq e^{n}
$$

Consequently, if $d<1$, then the above series converges for all complex $t$. If $d=1$, then the series converges for

$$
|t|<(a-s) / \mathrm{Ce} .
$$

If $d>1$, the dominating series diverges.

When the dominating series converges, $e^{A t} x$ is an analytic function and consequently we have

$$
\frac{d}{d t} e^{A t} x=A e^{A t} x
$$

that is, $x(t)=e^{A t} x$ is an analytic $X_{s}$-valued function satisfying

$$
\begin{aligned}
x^{\prime}(t) & =A x(t), \\
x(0) & =x .
\end{aligned}
$$

Moreover, if $y(t)$ is a continuous $X_{a}$-valued function, then

$$
x(t)=e^{A t} x+\int_{0}^{t} e^{A(t-\tau)} y(\tau) d \tau
$$

provides an $X_{s}$-valued solution of

$$
\begin{gathered}
x^{\prime}(t)=A x(t)+y(t) \\
x(0)=x
\end{gathered}
$$

for sufficiently small $t$. The verification of this last formula is left to the reader.

2. A linear theorem. Let us assume we have a scale $S$ on some interval $I$ and that $\delta$ is some positive number. Let us also assume that for each $t \in[0, \delta], A(t)$ is bounded operator on the scale $S$. We wish to consider the problem of finding $x(t)$ satisfying

$$
\begin{gathered}
x^{\prime}(t)=A(t) x(t)+y(t) \\
x(0)=x_{0},
\end{gathered}
$$

where $A(t), y(t), x_{0}$ satisfy the following conditions:

(I) There exists a constant $C$ such that if $\sigma, s \in I, \sigma<s, t \in[0, \delta]$, and $x \in X_{s}$, then 


$$
\|A(t) x\|_{\sigma} \leqq C(s-\sigma)^{-1}\|x\|_{s} .
$$

(II) If $\sigma, s \in I, \sigma<s$, then $A(t)$ is a continuous map of $[0, \delta]$ into $L\left(X_{s}, X_{\sigma}\right)$, i.e., $A(t) \in C\left[[0, \delta], L\left(X_{s}, X_{\sigma}\right)\right]$.

(III) $x_{0} \in X_{a}$ and $y(t) \in C\left[[0, \delta], X_{a}\right]$.

Theorem 2.1. Assume (I) and (II) hold. For some $\delta_{0}, 0<\delta_{0} \leqq \delta$, and every pair of data $x_{0}, y(t)$ such that (III) holds and for every $s \in I, s<a$, there is a function $x(t) \in C\left[\left[0, \delta_{0}(a-s)\right], X_{s}\right]$ satisfying (2.1).

THEOREM 2.2. Assume (I) and (II) hold. For every $\delta_{0}, 0<\delta_{0} \leqq \delta$, and every pair of data $x_{0}, f(t)$ satisfying (III), for every $s \in I, s \leqq a$, there is at most one function $x(t) \in\left[\left[0, \delta_{0}\right], X_{s}\right]$ and satisfying (2.1).

ProOF OF THEOREMS 2.1 AND 2.2. This proof is a generalization of the usual Picard iteration technique of ordinary differential equation theory. We wish to find a solution of (2.1) in the form

$$
x(t)=\sum_{k=0}^{\infty} x_{k}(t)
$$

where we will define $x_{k}$ inductively by

$$
\begin{gathered}
x_{0}(t)=x_{0}+\int_{0}^{t} y(\tau) d \tau \\
x_{k+1}(t)=\int_{0}^{t} A(\tau) x_{k}(\tau) d \tau, \quad k \geqq 0
\end{gathered}
$$

We will prove inductively that for all $s \in I, s<a$,

i) $x_{k}(t)$ is a continuous $X_{s}$-valued function, and

ii) $\left\|x_{k}(t)\right\|_{s} \leqq M(\operatorname{Cet} /(a-s))^{k}$.

For $k=0$, this is obvious. If we assume i) and ii) for $k=n$, then II implies $A(\tau) x_{k}(\tau)$ is a continuous $X_{s}$-valued function. Thus $x_{k+1}$ is well defined. Next

$$
\left\|x_{n+1}(t)\right\|_{s} \leqq \int_{0}^{t} C(\sigma-s)^{-1}\left\|x_{k}(\tau)\right\|_{\sigma} d \tau
$$

for any $\sigma, s<\sigma<a$. We choose

$$
\begin{gathered}
(\sigma-s)=\varepsilon(a-s) \\
(a-\sigma)=(1-\varepsilon)(a-s)
\end{gathered}
$$

and use the induction hypothesis to obtain

$$
\left\|x_{n+1}(t)\right\|_{s} \leqq M(a-s)^{-n-1} C^{n+1} e^{n}\left(t^{n+1} /(n+1)\right) \varepsilon^{-1}(1-\varepsilon)^{-n} .
$$

Note that the minimum of $1 /\left(\varepsilon(1-\varepsilon)^{n}\right)$ for $0 \leqq \varepsilon \leqq 1$ is given by $\varepsilon=$ $1 /(n+1)$ so that 


$$
\left\|x_{n+1}(t)\right\|_{s} \leqq M(\operatorname{Cet} /(a-s))^{n+1}(1-1 /(n+1))^{-n} / e .
$$

Noting that $(1+1 / n)^{n}<e$ completes the induction.

Thus we have for any $s<a, s \in I$ and $0 \leqq t \leqq \delta_{0}(a-s)$ where $\delta_{0}<$ $1 / \mathrm{Ce}$ that the series

$$
x(t)=\sum_{k=0}^{\infty} x_{k}(t)
$$

converges in $X_{s}$ uniformly in $t$. Thus $x(t)$ satisfies

$$
x(t)=x_{0}+\int_{0}^{t} A(\tau) x(\tau) d \tau+\int_{0}^{t} y(\tau) d \tau .
$$

This in turn implies that $x(t)$ is $C^{1}$ and satisfies the differential equation.

Finally, if there are two solutions $x_{1}(t)$ and $x_{2}(t)$ of $(2.1)$ then set $x(t)=$ $x_{1}(t)-x_{2}(t)$ and note that $x(t)$ satisfies

$$
x(t)=\int_{0}^{t} A(\tau) x(\tau) d \tau .
$$

If $x(t)=0$ for $0 \leqq t<\eta$, then by continuity $x(\eta)=0$. If $x(t)=0$ for $0 \leqq t \leqq \eta$, then

$$
x(t)=\int_{\eta}^{t} A(\tau) x(\tau) d \tau .
$$

Just as before we can prove that

$$
\|x(t)\|_{\sigma} \leqq M(C e(t-\eta) /(s-\sigma))^{n}
$$

for any $\sigma<s, \sigma \in I$. Thus $x(t)=0$ for $t \in[0, \eta+\varepsilon]$ for some $\varepsilon$. Consequently $x(t)=0$ wherever $x(t)$ is defined.

There are many generalizations of the above material. First it is possible to generalize the continuity in $t$ conditions to integrable in $t$ conditions (this is not recorded in the literature). It is also possible to study problems with singularities at $t=0$ (see Baouendi and Goulaouic [1]). There has also been an extensive study of nonlinear problems (see Baouendi and Goulaouic [3] and Nirenberg [22]).

3. A linear Cauchy-Kowalewski theorem. We shall prove a general existence and uniqueness theorem for the initial value problem for a first order system of partial differential equations. We use the standard multiindex notation

$$
z=\left(z_{1}, z_{2}, \ldots, z_{n}\right), \text { etc. }
$$

and all functions will be complex valued.

We wish to solve the initial value problem 


$$
D_{t} f(t, z)=\sum_{j=1}^{n} A_{j}(t, z) D_{j} f(t, z)+A_{0}(t, z) f(t, z)+g(t, z)
$$

$$
f(0, z)=f_{0}(z)
$$

for $f(t, z)$ when we are given $A_{j}, G$, and $f_{0}$.

Here we use the usual vector and matrix notat on for a system of P.D.E.'s:

$$
\begin{aligned}
f(t, z) & =\left(f_{1}(t, z), \ldots, f_{m}(t, z)\right) \\
g(t, z) & =\left(g_{1}(t, z), \ldots, g_{m}(t, z)\right) \\
f_{0}(z) & =\left(f_{10}(z), \ldots, f_{m 0}(z)\right)
\end{aligned}
$$

We assume that
(i) $A, f_{0}, g$ are analytic in $z$ for $|z|<a$, and
(ii) $A, g \in C\left[[0, \delta] \times \Delta_{a}, \mathbf{C}\right], \Delta_{a}=\{z,|z|<a\}$.

Note that assuming continuity and not analyticity in $t$ represents a major departure from the classical Cauchy-Kowalewski theorem. We introduce $A_{s}=\{f(z) ; f$ is continuous and bounded for $|z| \leqq s, f$ is analytic for $|z|$ $<s\} ;\|f\|_{s}=\sup _{|z| \leq s}|f(z)|$; and $C\left[[0, \delta], A_{s}\right]=\{f(t)=f(t, z) ; f$ is a continuous map of $[0, \delta]$ into $\left.A_{s}\right\}$.

Our assumptions imply that for all $b<a$

$$
\begin{aligned}
& \text { (i) } g(t)=g(t, z) \in C\left[[0, \delta], A_{0}^{m}\right] \\
& \text { (ii) } f_{0}=f_{0}(z) \in A_{b}^{m} \\
& \text { (iii) } A_{j}(t, z) \in C\left[[0, \delta], A_{b}^{m^{2}}\right] .
\end{aligned}
$$

TheOREM 3.1. For some $\delta_{0}, 0<\delta_{0} \leqq \delta$, and every pair of data $g$ and $f_{0}$ satisfying (3.5) and for every $s \in I, s<b$, there is a unique function $f(t) \in$ $C^{1}\left[\left[0, \delta_{0}(b-s)\right], A_{s}\right]$ satisfying (3.2).

Proposition 3.1. If $0<\sigma<s$ and $f \in A_{s}$, then there exists a constant C such that

$$
\left\|D_{j} f\right\|_{\sigma} \leqq C(s-\sigma)^{-1}\|f\|_{s} .
$$

If $g \in A_{a}, f \in A_{s}$ and $0<s \leqq a$, then

$$
\|g f\|_{s} \leqq\|g\|_{a}\|f\|_{s} .
$$

Proof. The first estimate is the Schwarz-Pick lemma and the second is trivial.

Proof of theorem 3.1. We choose any $a^{\prime}, b, b<a^{\prime}<a$, and set $I=$ $\left(0, a^{\prime}\right)$ and 


$$
X_{s}=\prod_{i=1}^{m} A_{s}=A_{s}^{m} .
$$

If $s<a^{\prime}$, then the second estimate of Proposition 3.1 implies that

$$
\left\|A_{j}(t, z) f(z)\right\|_{s} \leqq C \sup _{\substack{1 \leq k, t \leq m \\ 0 \leq t \leq \delta}}\left\|A_{j k r}(t, z)\right\|_{a^{\prime}}\|f\|_{s}
$$

and

$$
\begin{aligned}
& \left\|\left(A_{j}(t, z)-A_{j}(\tau, z)\right) f(z)\right\|_{s} \\
& \quad \leqq C \sup _{\substack{\leq \leq k, t \leq m \\
0 \leq t \leq \delta}}\left\|A_{j k}(t, z)-A_{j k r}(\tau, z)\right\|_{a^{\prime}}\|f(z)\|_{s}
\end{aligned}
$$

for some constant $C$. If we set

$$
A(t)=\sum_{j=1}^{n} A_{j}(t, z) D_{j}+A_{0}(t, z),
$$

then the above estimates and the first estimate of Proposition 3.1 imply that for all $\sigma<s<a^{\prime}$ and all $f \in X_{s}$ we have

$$
\|A(t) f\|_{\sigma} \leqq C(s-\sigma)^{-1}\|f\|_{s}
$$

and $A(t)$ maps $[0, \delta]$ continuously into $L\left(X_{s}, X_{\sigma}\right)$ for $0<\sigma<s \leqq a$. This verifies conditions (I) and (II) of Theorems 2.1 and 2.2, while condition (III) is given in (i) above.

4. Functorial remark. In this section we wish to construct new scales from old scales by passing to dual spaces. Let

$$
X_{s}^{*}=L\left(X_{s}, \mathbf{C}\right) \text {. }
$$

The following diagram is helpful in understanding our results $(\sigma<s)$.

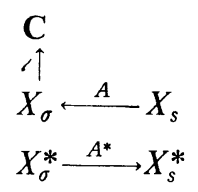

The definition of $A^{*}$ is

$$
A^{*}(\iota)=\iota \circ A
$$

for all $\ell \in X_{\sigma}^{*}$.

As before we can think of $X_{s}$ being injected into $X_{\sigma}$ for $\sigma<s$ and that leads to the following diagram.

$$
\begin{aligned}
& X_{s} \stackrel{i}{\stackrel{i}{\longrightarrow}} X_{\sigma} \\
& X_{s}^{*} \stackrel{i^{*}}{\longleftarrow} X_{\sigma}^{*}
\end{aligned}
$$


In order to view $i^{*}$ as an injection, we need $i^{*}$ to be one to one. Thus we make the following assumption.

Assumption 4.1. $X_{\text {s }}$ is dense in $X_{\sigma}$ for all $\sigma, s \in I, \sigma \leqq s$.

Proposition 4.1. Using the above definitions we have:

i) $i$ has dense range $\Leftrightarrow i^{*}$ is $1-1$,

ii) $i$ is $1-1 \Leftrightarrow i^{*}$ has weak ${ }^{*}$ dense range.

If $X_{s}$ is reflexive we may replace weak* dense by dense.

This is a standard application of the Hahn-Banach theorem.

Proposition 4.2. If $\left\{X_{s}, I\right\}$ is a scale, $m(s)$ is a finite valued monotonically decreasing function on $I$ and $I^{*}=m(I)$, then $\left\{X_{m(s)}^{*}, I^{*}\right\}$ is a scale. If $m$ is continuously differentiable with $m^{\prime} \leqq-M$ for some constant $M>0$ and $A$ is of type $d$ on $\left\{X_{s}, I\right\}$, then $A^{*}$ is of type $d$ on $\left\{X_{m(s)}^{*}, I^{*}\right\}$.

Proof. This is an easy exercise.

In the following we set $Z_{s}=X_{m(s)}^{*}, I^{*}=m(I), m(a)=a-s$ for some a.

TheOREM 4.1. Theorems 2.1 and 2.2 remain valid when $x, y, A(t), X_{s}$ and $I$ are replaced by $\mu, \nu, A^{*}(t), Z_{s}=X_{m(s)}$ and $I^{*}=m(I)$. That is, we can solve

$$
\begin{gathered}
\mu^{\prime}(t)=A^{*}(t) \mu(t)+\nu(t) \\
\mu(0)=\mu_{0}
\end{gathered}
$$

in the dual scale $S^{*}$.

Proof. This is also easy.

Example. As in Section 2, let $X_{s}=\{f ; f$ is analytic for $|z|<s, f$ is bounded and continuous for $|z| \leqq s\}$. We note that $\mu \in X_{s}^{*}$ if and only if

$$
\mu=\sum_{n=0}^{\infty} a_{n} \delta^{(n)}
$$

with certain conditions on $a_{n}$. We leave finding these conditions as an exercise. Recall that

$$
\begin{aligned}
\left\langle\delta^{n}, f\right\rangle & =(-1)^{n}(d / d z)^{n} f(0)=(-1)^{n} f\left({ }^{(n)}(0)\right. \\
f^{n}(0) & =(n ! / 2 \pi i) \int_{|z|=s}\left(f(\xi) / \xi^{n+1}\right) d \xi
\end{aligned}
$$

It is also true that $\mu$ has a Fourier transform 


$$
\begin{aligned}
\hat{\mu}(\xi) & =\left\langle\mu(z), e^{z \xi}\right\rangle \\
& =\sum_{n=0}^{\infty} \xi^{n}\left\langle\mu, z^{n}\right\rangle / n ! .
\end{aligned}
$$

It turns out that $\hat{\mu}(\xi)$ is an entire function of $\xi$. What is its order and type? We also note that the $\left\langle\mu, z^{n}\right\rangle$ are called the moments of $\mu$. Finally, if $g$ is an integrable function on $|z| \leqq s$, then $\mu_{g}$ defined by

$$
\left\langle\mu_{g}, f\right\rangle=\int_{|z| \leqq s} g(z) f(z) d z d \bar{z}
$$

belongs to $X_{s}^{*}$. For additional information about this and other types of generalized function spaces the book Topological Vector Spaces, Distributions and Kernels by Francois Treves [44] is an excellent reference.

5. Holmgren's uniqueness theorem. We present here an elementary uniqueness theorem of Holmgren type. For more extensive results, see [7, $33,47]$. We use the notation of section 3 but change condition (3.4) to

(i) $A$ is analytic in $z$ for $|z|<a$,

(ii) $f_{0} \in C\left[\Delta_{a}, \mathbf{C}\right]$, and

(iii) $A, g \in C\left[[0, \delta] \times \Delta_{a}, \mathbf{C}\right]$.

Thus we have dropped the condition that $f_{0}$ and $g$ be analytic.

THEOREM 5.1. If $f^{(i)}, i=1,2$, satisfy

(i) $f^{(i)}(t, z) \in C^{1}\left[\mathbf{R} \times \mathbf{C}^{n}, \mathbf{C}\right]$

(ii) for some $b<a$ and for each $t, 0 \leqq t \leqq \delta, i=1,2$, support $\left(f^{(i)}(t, z)\right) \subseteq \Delta_{b}$

(iii) for $i=1,2 f^{(i)}$ satisfies (3.2) with conditions (3.4) replaced by (5.1), then $f^{(1)}(t, z)=f^{(2)}(t, z)$ for all $z$ and $0 \leqq t \leqq \delta$.

Proof. First we note that $h=f^{(1)}-f^{(2)}$ satisfies (3.2) with $f_{0} \equiv g \equiv 0$. Because of (i), (ii), we see that $h(t)=h(t, z) \in C\left[[0, \delta], X_{b}^{*}\right]$ (see 4.9). The Uniqueness Theorem 2.2 implies that $h \equiv 0$.

6. More scales of Banach spaces. In this section we wish to give some additional examples of scales of Banach spaces. These examples are typical of the types of scales in use at the present time. In a later section we will introduce more general scales and show that in some sense scales are not sensitive to the particulars of their description and that the scales are intrinsic to the situation in which they are used. Thus one should take the view that in the following we are making convenient and somewhat arbitrary choices. We will usually attempt to make our scales consist of 
Banach algebras or Hilbert spaces. When we introduce a scale, we always attempt to do the following:

(1) Give a classical description of the scale;

(2) Give a classical description of the dual scale;

(3) Estimate the operator norm of differentiation acting on the scale;

(4) Describe the multipliers on the scale.

In our first example we wish to describe certain scales of entire or analytic functions of one complex variable which will depend on two real parameters $d, 0 \leqq d \leqq 1$, and $s, 0<s<\infty$. If $f$ is analytic near $z=0$, then

$$
f(z)=\sum_{n=0}^{\infty} f_{n} z^{n} / n !
$$

We define

$$
\begin{aligned}
\|f\|_{d, s}^{2} & =\sum_{n=0}^{\infty}\left(\left|f_{n}\right| s^{n} / n !^{d}\right)^{2} \\
E_{d, s} & =\left\{f,\|f\|_{d, s}<\infty\right\} .
\end{aligned}
$$

Proposition 6.1. For $d$ fixed, $0 \leqq d \leqq 1,\left\{E_{d, s},(0, \infty)\right\}$ is a scale in the parameter $s$. Let

$$
k=1 /(1-d), 0 \leqq d<1 .
$$

If $0 \leqq d<1$ and $f \in E_{d, s}$, then $f(z)$ is entire and there is a constant $C$ such that if $t$ satisfies

$$
t k s^{k}>1
$$

then

$$
|f(z)| \leqq C \exp \left(t|z|^{k}\right) .
$$

Conversely, if $0 \leqq d<1, f(z)$ is entire, and there is a constant $C$ such that

$$
|f(z)| \leqq \exp \left(t|z|^{k}\right)
$$

and s satisfies

$$
t k s^{k}<1,
$$

then $f \in E_{d, s}$. If $d=1$ and $f \in E_{d, s}$, then $f$ is analytic for $|z|<s$. Conversely, iff is analytic for $|z|<t$ for some $t>s$, then $f \in E_{d, s}$.

Proof. The proof that $E_{d, s}$ is a scale is straightforward. Note that the set $\left\{z^{n}, 0 \leqq n<\infty\right\}$ is dense in $E_{d, s}$.

We need to know that for all $r, t \in R^{+}, n, k \in N^{+}$, we have

$$
\min _{r>0}\left\{r^{-n} \exp \left(t r^{k}\right)\right\}=(\mathrm{tke} / \mathrm{n})^{n / k}
$$


and

$$
M^{-1} n^{n} \leqq n ! e^{n} n^{-1 / 2} \leqq M n^{n}
$$

for some constant $M>0$. The equality is a calculus exercise and the inequality is Stirling's formula.

If $f \in E_{d, s}$, and $r=|z|$, then

$$
\begin{aligned}
|f(z)| & \leqq \sum_{n} r^{n}\left|f_{n}\right| / n ! \\
& \leqq\left(\sum_{n}\left(\left|f_{n}\right| s^{n} / n ! d\right)^{2}\right)^{1 / 2}\left(\sum_{n}\left(r^{n} s^{-n} n !^{(d-1)}\right)^{2}\right)^{1 / 2} \\
& \leqq\|f\|_{d, s} \exp \left(t r^{k}\right)\left(\sum_{n}\left(\left(s^{k} \mathrm{kee}\right)^{-n}\left(n^{n} / n !\right)^{1 / k}\right)^{2}\right)^{1 / 2} .
\end{aligned}
$$

Stirling's formula completes the estimate. On the other hand, if $|f(z)| \leqq C$ $\exp \left(t r^{k}\right)$ and $r=|z|$, then Cauchy's integral theorem implies

$$
\left|f_{n}\right|=\left(\frac{d}{d z}\right)^{n} f(0) \mid \leqq n ! r^{-n} \exp \left(t r^{k}\right) .
$$

If we minimize over $r$ and apply Stirling's formula, we obtain

$$
\begin{aligned}
\left|f_{n}\right| & \leqq C(\mathrm{tke} / n)^{n / k} n ! \\
& \leqq C s^{-n} n !^{d}\left(s^{k} t k\right)^{n / k}\left(e n ! / n^{n}\right)^{1 / k} .
\end{aligned}
$$

Summing this gives the result.

We leave as an exercise whether or not $f \in E_{1, s}$ can be characterized by its behavior on $|z|=s$.

Proposition 6.2. If $0 \leqq d \leqq 1,0<\varepsilon<1$ and $\varepsilon \leqq \sigma<s \leqq 1 / \varepsilon$, then

$$
\begin{aligned}
\|D f\|_{\sigma} & \leqq C(\varepsilon)(s-\sigma)^{-d}\|f\|_{s} \\
\|z f\|_{\sigma} & \leqq C(\varepsilon)(s-\sigma)^{d-1}\|f\|_{s} .
\end{aligned}
$$

Here, $C(\varepsilon)$ is a constant independent of $s$ and $\sigma$, and $\|\cdot\|_{s}=\|\cdot\|_{d, s}$.

Proof. First we note that calculus implies that

$$
\sup _{n \geqq 0}\left(r^{n} n^{d}\right) \leqq(d / e)^{d}(\ln (1 / r))^{-d}
$$

for $0 \leqq r<1$ and $d>0$. Also

$$
1 / \ln (1 / r) \leqq(1 /(1-r)) \text {. }
$$

Now we compute

$$
D f=\sum_{n=1}^{\infty} n f_{n} z^{n-1} / n !=\sum_{n=0}^{\infty} f_{n+1} z^{n} / n !
$$




$$
\begin{aligned}
\|D f\|_{\sigma} & =\left(\sum_{n=0}^{\infty}\left(\left|f_{n+1}\right| \sigma^{n} / n !^{d}\right)^{2}\right)^{1 / 2} \\
& =\left(\sum_{n=0}^{\infty}\left(\left|f_{n+1}\right| s^{n+1} /(n+1) ! !^{d}\right)^{2}\right)^{1 / 2} \sup _{n \geqq 0}\left\{\left(\sigma^{n} / s^{n+1}\right)(n+1)\right\} \\
& \leqq \sigma^{-1}(s d / e)^{d}(s-\sigma)^{-d}\|f\|_{s},
\end{aligned}
$$

$$
\begin{gathered}
z f=\sum_{n=0}^{\infty} f_{n} z^{n+1} / n !=\sum_{n=1}^{\infty} n f_{n-1} z^{n} / n ! \\
\|z f\|_{\sigma}=\left(\sum_{n=1}^{\infty}\left(n\left|f_{n-1}\right| / \sigma^{n} / n ! d\right)^{2}\right)^{1 / 2} \\
=\left(\sum\left(\left|f_{n-1}\right| s^{n-1} /(n-1) ! d\right)^{2}\right)^{1 / 2} \sup _{n \geq 0}\left\{\left(\sigma^{n} / s^{n-1}\right) n^{1-d}\right\} \\
\leqq s^{-1}(s(1-d) / e)^{1-d}(s-\sigma)^{d-1}\|f\|_{s} .
\end{gathered}
$$

It is not known whether or not there exist nontrivial functions $g(z)$ such that

$$
\|g f\|_{s} \leqq C\|f\|_{s},
$$

that is; are there multipliers of type zero.

We are now left with the problems of describing the dual scale $E_{d, s}^{*}$. To motivate our descriptions we note that if

$$
f=\sum f_{n} z^{n} / n !
$$

and $\mu$ is a linear functional, then, at least formally,

$$
\mu(f)=\sum_{n=0}^{\infty} f_{n} \mu\left(z^{n}\right) / n !
$$

For any analytic functional we define the $n$th moment of $\mu$ by

$$
\mu_{n}=\left\langle\mu, z^{n}\right\rangle .
$$

We will also define the Fourier-Borel transform of $\mu$ by

$$
\hat{\mu}(\xi)=\mu\left(e^{z \xi}\right)=\sum \mu\left(z^{n}\right) \xi^{n} / n !
$$

if either expression is well defined.

Proposition 6.3. If $0 \leqq d \leqq 1,0<s<\infty$, then

$$
\mu \in E_{d, s}^{*}
$$

if and only if

$$
\hat{\mu}(\xi) \in E_{1-d, 1 / s}
$$


Moreover

$$
\begin{gathered}
\hat{\mu}(\xi)=\sum \mu_{n} \xi^{n} / n ! \\
\mu=\sum(-1)^{n} \mu_{n} \delta^{(n)} / n ! \\
\langle\mu, f\rangle=\mu(f)=\sum f_{n} \mu_{n} / n !
\end{gathered}
$$

Proof. First we note that if $f \in E_{d, s}$, then

$$
f(z)=\sum f_{n} z^{n} / n !, f_{n}=D^{n} f(0)
$$

in the sense that the series converges to $f$ in $E_{d, s}$. Thus if $\mu \in E_{d, s}^{*}$, then

$$
\mu(f)=\sum f_{n} \mu_{n} / n !, \mu_{n}=\mu\left(z^{n}\right) .
$$

If we set

$$
\langle\mu, f\rangle=\sum \mu_{n} f_{n} / n !
$$

which is a weighted inner product, then we can view the duality between $E_{d, s}$ and $E_{d, s}^{*}$ as a weighted duality between sequences. Thus the $t_{2}$ duality implies that $\mu \in E_{d, s}^{*}$ if and only if

$$
\sum\left(\left|\mu_{n}\right| s^{-n} n !^{d-1}\right)^{2}<\infty,
$$

that is if and only if

$$
\hat{\mu} \in E_{1-d, 1 / s} .
$$

Moreover

$$
\|\mu\|_{d, s}=\|\hat{\mu}\|_{1-d, 1 / s} .
$$

This equality implies that the series

$$
\sum(-1)^{n} \mu_{n} \delta^{(n)} / n !
$$

converges in $E_{d, s}^{*}$. Finally, we note that if

$$
\tilde{\mu}=\mu-\Sigma(-1)^{n} \mu_{n} \delta^{(n)} / n !,
$$

then $\tilde{\mu}\left(z^{n}\right)=0$, and because $z^{n}$ is dense in $E_{d, s}$, we have $\tilde{\mu}=0$.

In our next example we wish to give spaces that correspond to our previous spaces when $d>1$. For a discussion of these types of spaces (called Gevrey spaces) including the fact that these spaces contain compactly supported functions and thus are not quasi-analytic, see Gel'fand and Shilov [12 v. 2] or Lions and Magenes [79]. Although we are most interested in these spaces when $d>1$, we note that the definitions make sense for $d \geqq 0$ and give a slightly different version of the previous spaces when $d \leqq 1$.

Let $d \geqq 0,0<s<\infty, \Omega \cong R$ and $f \in C^{\infty}(\Omega)$, and then define 


$$
\begin{gathered}
\|f\|^{2}=\int_{Q}|f(x)|^{2} d x, L_{2}=\{f,\|f\|<\infty\} \\
\|f\|_{d, s}^{2}=\sum\left(\left\|D^{n} f\right\| s^{n} / n ! !^{d}\right)^{2} \\
G_{d, s}=\left\{f,\|f\|_{d, s}<\infty\right\} .
\end{gathered}
$$

Proposition 6.4. If $d>1$ and $0<s<\infty$, then $G_{d, s}$ is a scale in $s$. Moreover, there is a set $E$ of functions such that $E$ is dense in $G_{d, s}$ for all $s$.

Proof. All is straightforward except for the density of $E$. First note that there exists a function $g(d, s, \xi)$ such that

$$
\|f\|_{d, s}^{2}=\int|\hat{f}(\xi)|^{2} g(d, s, \xi) d \xi,
$$

If $\chi$ is the characteristic function of $\Omega$, then we can take the Fourier transform $\hat{E}$ of $E$ to be $\hat{E}=\left\{f(\xi) ; f(\xi)=\chi(\xi) * g(\xi)\right.$ for all $\left.g \in C_{0}^{\infty}(R)\right\}$ where $*$ is convolution.

Let us consider the problem of multipliers. For this we need Leibniz's formula

$$
D^{n}(g f)=\sum_{k=0}^{\infty}\left(\begin{array}{l}
n \\
k
\end{array}\right)\left(D^{k} g\right)\left(D^{n-k} f\right) .
$$

We also need the definitions

$$
\begin{gathered}
\|g\|_{\infty}=\sup _{x \in \Omega}|f|, L_{\infty}=\left\{g ;\|g\|_{\infty}<\infty\right\} \\
\|g\|_{d, s}=\sum_{n=0}^{\infty}\left(\left\|D^{n} g\right\|_{\infty} s^{n} / n ! !^{d}\right) \\
M G_{d, s}=\left\{g ;\|g\|_{d, s}<\infty\right\} .
\end{gathered}
$$

Proposition 6.5. If $d>1,0<s<\infty, f \in G_{d, s}$ and $g \in M G_{d, s}$, then

$$
\|g f\|_{d, s} \leqq\|g\|_{d, s}\|f\|_{d, s} .
$$

If $d \geqq 0,0<\varepsilon<1, \varepsilon \leqq \sigma<s \leqq 1 / \varepsilon$, and $f \in G_{d, s}$, then there exists $a$ constant $C(\varepsilon)$ such that

$$
\|D f\|_{\sigma} \leqq C(\varepsilon)(s-\sigma)^{-d}\|f\|_{s} .
$$

Proof. We calculate:

$$
\begin{aligned}
\|g f\|_{d, s} & \left.\leqq\left(\sum_{n=0}^{\infty}\left(\begin{array}{l}
n \\
k
\end{array}\right)\left\|D^{n-k} g D^{k} f\right\| s^{n} / n !^{d}\right)^{2}\right)^{1 / 2} \\
& \leqq\left(\sum_{n=0}^{\infty}\left(\sum_{k=0}^{n}\left(\begin{array}{l}
n \\
k
\end{array}\right)^{1-d}\left\|D^{n-k} g\right\|_{\infty} S^{n-k}(n-k) !^{-d}\left\|D^{k} f\right\| s^{k} k !^{-d}\right)^{2}\right)^{1 / 2} \\
& \leqq\|g\|_{d, s}\|f\|_{d, s} .
\end{aligned}
$$


The proof of the inequality for differentiation is given in Proposition 5.3.

It is not known whether or not we can modify $G_{d, s}$ so that it becomes a Banach algebra.

II. General theory. 0. Introduction. The purpose of this section is to give general versions of the results in the previous section.

Our first project will be to set up a general linear theory where we replace the operator $d / d t$ in Theorem I.2.1 by a more general operator $D$, which we will call an evolution operator. In the applications, $D$ is generally a hyperbolic or parabolic operator. We give an example in Section 2. Also this particular version of our results was used to study the initial value problem for hyperbolic equations with multiple characteristics, see [33].

The second project will be to give an account of some nonlinear results. Finally we will give a general discussion of how to choose a scale of spaces in a given problem.

1. The linear theorem. In this section we wish to discuss problems of the form

$$
\begin{gathered}
d x(t) / d t-A(t) x(t)=B(t) x(t)+y(t) \\
x(0)=z .
\end{gathered}
$$

Thus, in terms of the previous material, we have replaced $(A(t)$ by $A(t)+$ $B(t)$. The idea is to assume that the problem

$$
\begin{gathered}
d x(t) / d t-A(t) x(t)=0 \\
x(0)=z
\end{gathered}
$$

is well posed in the classical sense and then perturb this well posed problem with some operator $B(t)$ so that (1.1) is not well posed in the classical sense. In the applications we see that $A(t)$ and $B(t)$ are differential or pseudo-differential operators. For this theory to give better results than the previous material, $B(t)$ must be of lower order than $A(t)$. In general both $A(t)$ and $B(t)$ are unbounded as operators on any individual space $X_{s}$. We give our results for systems because the extension to systems is not straightforward.

We introduce the notion of an evolution operator as a way of saying that (1.2) is well posed. However, before we do this we need to set up a few things.

Definition 1.1. A real valued function $r(t)$ on the interval $0 \leqq t \leqq \delta$ will be called a scale function if it satisfies

a) $r(0)=1, \quad r(t)>0$ 
b) $r(t)$ is continuously differentiable

c) $r^{\prime}(t) \leqq 0$.

Definition 1.2. Let $\delta>0, S=\left\{X_{s}, I\right\}$ be a scale, $r(t)$ be a scale function on $[0, \delta], k \in N^{+}$and $s \in I$. Then we will say $x(t) \in C\left[\delta, X_{s}, r\right]$ if there exists a constant $C$ such that

$$
x(t) \in C\left[[0, \eta], X_{s r(\eta)}\right] \text { and }\|x(t)\|_{s r(t)} \leqq C
$$

for all $\eta, 0 \leqq \eta \leqq \delta$, such that $\operatorname{sr}(\eta) \in I$. Also $x(t) \in C^{k}\left[\delta, X_{s}, r\right]$ if $(d l d t)^{j} x(t) \in C\left[\delta, X_{s}, r\right]$ for all $j, 0 \leqq j \leqq k$.

Proposition 1.1. If $x(t) \in C\left[\delta, X_{s}, r\right]$, then $g(t)=\|x(t)\|_{s r(t)}$ is a measurable function of $x$ on $[0, \delta]$.

Proof. For $\tau<t$ we have

$$
\|x(t)\|_{s r(t)}-\|x(\tau)\|_{s r(\tau)} \leqq\|x(t)-x(\tau)\|_{s r(t)}
$$

which implies that $g(t)$ is left lower semi-continuous and thus measurable.

Definition 1.3. Let $\delta>0, S=\left\{X_{s}, I\right\}$ be a scale, $r(t)$ be a scale function, and for each $t \in[0, \delta]$ let $A(t)$ be a bounded operator on $S$. If $A(t)$ satisfies:

(I) There are positive constants $C$ and $d$ such that if $\sigma, s \in I, \sigma<s$, $t \in[0, \delta], x \in X_{s}$, then

$$
\|A(t) x\|_{\sigma} \leqq C(s-\sigma)^{-d}\|x\|_{s} ;
$$

(II) For all $\sigma, s \in I, \sigma<s, A(t) \in C\left[[0, \delta], L\left(X_{s}, X_{\sigma}\right)\right]$;

(III) There exists $C>0$ such that if $s \in I$ and $y(t) \in C\left[\eta, X_{s}, r\right]$ for some $\eta \leqq \delta$, then there exists a unique $x(t) \in C^{1}\left[\eta, X_{\sigma}, r\right]$ for all $\sigma \in I, \sigma<s$, satisfying

$$
\begin{gathered}
d x(t) / d t-A(t) x(t)=y(t) \\
x(0)=0
\end{gathered}
$$

and

$$
\|x(t)\|_{\sigma r(t)} \leqq C \int_{0}^{t}\|y(\tau)\|_{\sigma r(\tau)} d \tau
$$

for $0 \leqq t \leqq \delta$, and $\sigma r(t) \in I$

then $D=\partial / \partial t-A(t)$ is said to be an evolution operator.

Remark. If in Definitions $1.1,1.2,1.3, I$ is replaced by $I^{\prime} \leqq I$, then the definitions still hold. 
We are now in a position to state our initial value problem. We wish to solve

$$
\begin{gathered}
\left(d / d t-A_{i}(t)\right) x_{i}(t)=\sum_{i=1}^{N} B_{i, j}(t) x_{j}(t)+y_{i}(t) \\
x_{i}(0)=z_{i}
\end{gathered}
$$

for $x_{i}(t), 1 \leqq i \leqq N$. We will need the following conditions (here $\delta>0$ is fixed and $S=\left\{X_{s}, I\right\}$ is a scale).

(I) For $1 \leqq i \leqq N,\left(d / d t-A_{i}(t)\right)$ are evolution operators on $S$ with common scale function $r(t)$.

(II) There exists $N$ numbers $p_{i}$ and constant $C_{i, j}$ such that if $\sigma, d \in I$, $\sigma<s$ and $x \in X_{s}$, then

$$
\left\|B_{i, j}(t) x\right\|_{\sigma} \leqq C_{i, j}(s-\sigma)^{-1-p_{i}+p_{j}}\left\|x_{s}\right\| .
$$

Also $B_{i, j}(t) \in C\left[[0, \delta], L\left(X_{s}, X_{\sigma}\right)\right]$.

(III) There exists $a \in I$ such that $z_{i} \in X_{a}$ and for all $s \in I, s<a, y(t) \in$ $C\left[\delta, X_{s}, r\right]$.

Remark. If $1+p_{i}-p_{j}<0$, then $B_{i, j} \equiv 0$. The concept of type can be generalized so as to avoid this restriction. However, we have no applications for this generalization, so we avoid it here.

REMARK. The condition III on $y(t)$ is implied by the simpler condition $y(t) \in C\left[\delta, X_{a}, r\right]$, which is in turn implied by the even simpler condition $y(t) \in C\left[[0, \delta], X_{a}\right]$. The stated condition is used because it is most appropriate to the methods used to prove the next theorem.

THEOREM 1.1. We assume system (1.3) satisfies conditions I, II, and III. For every $s<a, s \in I$, there exists $\eta=\eta(s) \leqq \delta$ and functions $x_{i}(t) \epsilon$ $C^{1}\left[\eta, X_{s}, r\right]$ satisfying (1.3). Conversely, if for some $\eta \leqq \delta$ and $s \in I, x_{i}(t)$, $\tilde{x}_{i}(t) \in C^{1}\left[\eta, X_{s}, r\right]$ and $x_{i}(t), \tilde{x}_{i}(t)$ satisfy $(1.3)$, then $x_{i}(t)=\tilde{x}(t)$ for $t$ sufficiently small.

Proof. We shall show that $x_{i}(t)=z_{i}+\sum_{n=0}^{\infty} x_{i}^{(n)}(t)$ is the solution of our problem where we define $x^{(n)}(t)$ inductively by

(i) $\left(d / d t-A_{i}(t)\right) x_{i}^{(0)}(t)=\tilde{y}_{i}(t)=y_{i}(t)+y_{i}(t)+A_{i}(t) z_{i}$

$$
x_{i}(0)=0
$$

(ii) $\left(d / d t-A_{i}(t)\right) x_{i}^{(n+1)}(t)=z_{i}^{(n)}(t)=\sum_{j=1}^{N} B_{i, j}(t) x_{j}^{(n)}(t)$

$$
x_{i}^{(n+1)}(0)=0
$$

for $n \geqq 0$. We first note that there are constants $C_{1}, C_{2}$ and $K^{\prime}$ such that 


$$
\begin{aligned}
\left\|B_{i, j}(t) x\right\|_{\sigma} & \leqq C_{1}(s-\sigma)^{-1-p_{i}+p_{j}\|x\|_{s}} \\
\left\|A_{i}(t) x\right\|_{\sigma} & \leqq C_{2}(s-\sigma)^{-d_{i}} \\
\|x(t)\|_{s r(t)} & \leqq K^{\prime} \int_{0}^{t}\|y(\tau)\|_{s r(\tau)} d \tau
\end{aligned}
$$

provided $\left(d / d t-A_{i}(t)\right) x(t)=y(t), x(0)=0$, for some $j, 1 \leqq i \leqq N$. In this and what follows we always have $\sigma<a, \sigma, s \in I$ and $0 \leqq t \leqq \delta$. Next, if $\operatorname{sr}(t) \in I$, then $\tilde{y}_{i}(t) \in C\left[\delta, X_{s}, r\right]$ and

$$
\begin{aligned}
\left\|\tilde{y}_{i}(t)\right\|_{s r(t)} & \leqq\left\|y_{i}(t)\right\|_{s r(t)}+C_{2}(a-s)^{-d_{i}}\left\|z_{i}\right\|_{a} \\
& \leqq K_{i}+C(a-s)^{-d_{i}}\left\|z_{i}\right\|_{a}
\end{aligned}
$$

where $K_{i}$ is given in the definition of $y_{i} \in C\left[\delta, X_{s}, r\right]$.

If we set

$$
M^{\prime}=\max _{i}\left(K_{i}+C(a-s)^{-d_{i}}\left\|z_{i}\right\|_{a}\right) K^{\prime},
$$

then because $d / d t-A_{i}$ is an evolution operator, we have

$$
x_{i}^{(0)}(t) \in C^{1}\left[\delta, X_{s}, r\right]
$$

and

$$
\left\|x_{i}^{(0)}(t)\right\|_{s r(t)} \leqq K^{\prime} \int_{0}^{t}\left\|\tilde{y}_{i}(\tau)\right\|_{s p(\tau)} d \tau \leqq M^{\prime} t
$$

for all $(s, t)$ such that $s r(t) \in I$.

We shall prove by induction that for all $(s, t)$ such that $s \in I, s<a$, $0 \leqq t \leqq \delta$ and $s r(t) \in I$ that

(i) $x_{i}^{(n)}(t) \in C^{1}\left[\delta, X_{s}, r\right]$

(ii) $\left\|x_{i}^{(n)}(t)\right\|_{s r(t)} \leqq M K^{n}(n+1)^{p_{i}}(r(t)(a-s))^{-n-p_{i}-p} t^{n+1}$ where $p=\max _{i}\left|p_{i}\right|, M=M^{\prime} \max _{i}\left(a^{p+p_{i}}\right)$ and $K$ is to be chosen later.

The above estimates give the case $n=0$; so we now do the induction step. Thus we have

$$
\begin{gathered}
\left\|z_{i}^{(n)}(t)\right\|_{s r(t)} \leqq M K^{n} t^{n+1} C_{1} \\
\cdot \sum_{j=1}^{n}((\sigma-s) r(t))^{-p_{i}+p_{j}(n+1)^{p_{j}}((a-\sigma) r(t))^{-n-p_{j}-p}}
\end{gathered}
$$

for any $\sigma, s<\sigma<a$. In particular, if $(\sigma-s)=(a-s) /(n+1),(a-\sigma)$ $=(a-s) /(1+1 / n), K=C_{1} e 4^{p} K^{\prime}$, then

$$
(1+1 / n)^{n+p_{j}+p} \leqq e 4^{p}
$$

and

$$
\left\|z_{i}^{(n)}(t)\right\| \leqq\left(K^{\prime}\right)^{-1} M K^{n+1}(r(t)(a-s))^{-1-n-p_{i}-p}(n+1)^{p_{i}+1} t^{n+1} .
$$


The continuity of $B_{i, j}(t)$ in $t$ and the above estimate imply that $z_{i}^{(n)}(t) \in$ $C\left[\delta, X_{s}, r\right]$. Consequently, there exist $x_{i}^{(n+1)}$ satisfying $x_{i}^{(n+1)}(t) \in$ $C\left[\delta, X_{s}, r\right]$ and

$$
\begin{aligned}
\left\|x_{i}^{(n+1)}(t)\right\|_{s(t)} & \leqq K^{\prime} \int_{0}^{t}\left\|z_{i}^{(n)}(\tau)\right\|_{s r(\tau)} d \tau \\
& \leqq M K^{n+1}(a-s)^{-1-n-p_{i}-p}(n+1)^{p_{i}+1} \int_{0}^{t} \tau^{n+1} r(\tau)^{-1-n-p_{i}-p} d \tau
\end{aligned}
$$

However $r(\tau) \geqq r(t)$, so that the integral is estimated by

$$
\left(t^{n+2} /(n+2)\right) r(t)^{-1-n-p_{i}-p}
$$

which gives the desired estimate.

The above estimate implies that $x_{i}^{n}(t)$ is summable in $X_{s p(t)}$ for $t / r(t) \leqq$ $(a-s) / K$, provided $\operatorname{sr}(t) \in I$. Because $r(t)$ is decreasing and $r(0)=1$, we can choose $\eta=\eta(s)$ depending on $s$ such that $0 \leqq t \leqq \eta$ implies $t / r(t)$ $\leqq \varepsilon(a-s) / K, \operatorname{sr}(t) \in I$, for any $\varepsilon, 0<\varepsilon<1$. Thus, if $0 \leqq t \leqq \eta$, then

$$
\left\|x_{i}^{(n)}(t)\right\|_{s r(t)} \leqq M^{\prime \prime}(n+1)^{p_{i} \varepsilon^{n}}
$$

for some constant $M^{\prime \prime}$. However,

$$
\left\|x_{i}^{(n)}(\tau)\right\|_{s r(t)} \leqq\left\|x_{i}^{(n)}(\tau)\right\|_{s r(\tau)} \leqq M^{\prime \prime}(n+1)^{p_{i} \varepsilon^{n}},
$$

which implies that $x_{i}^{(n)}(\tau)$ is summable in $X_{s r(t)}$, uniformly in $\tau, 0 \leqq \tau$ $\leqq t$, that is, $x_{i}(t) \in C\left[\eta, X_{s}, r\right]$. Next, because of (1.3) we see that for any $\sigma<s, 0 \leqq \tau \leqq t, d x_{i}^{(n)}(\tau) / d t$ is uniformly summable in $X_{\sigma r(t)}$, that is, $x_{i}(t) \in C^{1}\left[\eta, X_{\sigma}, r\right]$. However, for $\sigma$ sufficiently close to $s$ and $0 \leqq t \leqq \eta$, we have $t / r(t) \leqq \varepsilon^{\prime}(a-\sigma) / K$ for any $\varepsilon^{\prime}, \varepsilon<\varepsilon^{\prime}<1$. Clearly $x_{i}(t)$ satisfy the differential equation (1.3). If we replace $\sigma$ by $s$, we have the existence part of our result.

Because of linearity, the problem of uniqueness can be reduced to showing that if $z_{i}=0, y_{i}(t)=0$ and for some $\eta \leqq \delta$ and $s \in I, x_{i}(t) \in$ $C\left[\eta, X_{s}, r\right]$, then $x_{i}(t)=0$ for $t$ sufficiently small.

In essentially the same way we obtain our estimates above, we can obtain the following result. If $\sigma<s, \sigma \in I, t \in[0, \eta]$, and $\sigma r(t) \in I$, then

$$
\left\|x_{i}(t)\right\|_{s r(t)} \leqq K^{n}(n+1)^{p_{i}}(r(t)(s-\sigma))^{-n-p_{i}-p^{n+1}} t^{n+} .
$$

Thus, for all $t$ such that $t / r(t)<(s-\sigma) / K, \sigma r(t) \in I$, for some $\sigma \in I$, we obtain $x_{i}(t)=0$. For any $\sigma<s$, the above inequalities hold for $t$ sufficiently small. This implies the uniqueness.

2. Example Let us consider a non-skewadjoint perturbation of the Schroedinger equation 


$$
\begin{gathered}
\partial f / \partial t-i \partial^{2} f / \partial x^{2}=c \partial f / \partial x+g \\
f=f(t)=f(x, t), g=g(t)=g(x, t) \\
f(x, 0)=f_{0}(x) .
\end{gathered}
$$

Here $i^{2}=-1, c$ is a complex number and $f_{0}(x)$ and $g(x, t)$ are given complex valued functions of $x \in \mathbf{R}, t \in[0, \delta]$. If $c$ were real, then

$$
i \partial^{2} / \partial x^{2}+c \partial / \partial x
$$

would be formally skewadjoint and it would be reasonable to use the theory of semi-groups to find a solution in $L_{2}$ (and in fact this has been done). If $c$ is not real, then this is not reasonable. In fact, using the Fourier transform, one can easily see that the equation does not, in general, possess $L_{2}$ solutions for $f_{0}$ in $L_{2}$ and $g(x, t)=0$ when $c$ is not real.

We now apply the previous theory to this example. It is easily seen that these results agree with the Fourier transform results. However the abstract propagator theory can handle much more general situations and the Fourier transform techniques cannot.

In this elementary situation we can take $r(t)=1$,

$$
\begin{gathered}
D=\partial / \partial t-i \partial^{2} / \partial x^{2}, \\
X_{s}=G_{d, s}, d=1, \\
I=(0, \infty)
\end{gathered}
$$

where $G_{d, s}$ was given in (16.34). The classical results for the Schroedinger equation imply that if

$$
\begin{gathered}
D f(t)=g(t), f(t)=f(x, t), g(t)=g(x, t) \\
f(0)=0)
\end{gathered}
$$

and $g(t) \in C\left[[0, \delta], L_{2}\right]$ (is a continuous mapping of $[0, \delta]$ into $L_{2}$ ), then there exists $f(t) \in C^{1}\left[[0, \delta], L_{2}\right]$ such that

$$
\|f(t)\| \leqq \int_{0}^{t}\|g(\tau)\| d \tau .
$$

This easily implies that

$$
\|f(t)\|_{s} \leqq \int_{0}^{t}\|g(\tau)\|_{s} d \tau
$$

provided $g \in C\left[[0, \delta], X_{s}\right]$. Note that $C\left[\delta, X_{s}, r\right]=C\left[[0, \delta], X_{s}\right]$ when $r(t)$ $\equiv 1$. It is now clear that $D$ is an evolution operator on $S$. Next, Proposition (16.5) implies that for some constant $C>0$ that

$$
\|c \partial f / \partial x\|_{\sigma} \leqq C(s-\sigma)^{-1}\|f\|_{s}
$$


if we make the additional restriction that $I=(\varepsilon, 1 / \varepsilon)$ where $0<\varepsilon<1$.

Proposition 2.1. If for some $\delta>0, a>0, g(t)=g(x, t) \in C[[0, \delta]$, $\left.X_{a}\right], f_{0}=f_{0}(x) \in X_{a}$, then for every $s, 0<s<a$, there exists $\eta=\eta(s)$ and a function $f(t) \in C^{1}\left[[0, \eta], X_{s}\right]$ satisfying (2.1). Conversely, if $f(t), \tilde{f}(t)$ $\in C^{1}\left[[0, \eta], X_{s}\right.$ and satisfy $(1.3)$, then $f(t)=\tilde{f}(t)$ for $t$ sufficiently small.

Proof. This is an immediate consequence of Theorem II1.1 and the above remarks.

Remark. This result is not a consequence of the classical semigroup theore or the Cauchy-Kowalewski theory. As pointed out before, these results can be obtained using the Fourier transform. However, if any of the coefficients of the problem were to depend on the variable $x$, then the Fourier transform would not help. It is still unknown to which class of equations with variable coefficients this version of the abstract propagator theory applies. We also note that if $\partial^{2} / \partial x^{2}$ has a coefficient that depends on $x$, then one can no longer take $r(t) \equiv 1$. For much deeper results in this direction see [45].

3. Nonlinear theorems. In this seciton we will study a nonlinear Cauchy problem of the form

$$
\begin{aligned}
d x(t) / d t & =F(x(t), t) \\
x(0) & =0
\end{aligned}
$$

for $x(t) \in S=\left\{X_{s}, I\right\}$ where $S$ is a scale.

REMARK. There are several nonlinear versions of Theorem I.2.1. in the literature. In general they assume $F(x, t)$ is Lipschitz continuous in $x$ and is globally defined in $x$. It is a major technical difficulty to weaken these conditions and have $F(x, t)$ defined only locally.

For some fixed $\eta>0$ and $R>0$, let

$$
B(s, R)=\left\{x \in X_{s},\|x\|_{s}<R\right\} .
$$

We will always assume the following conditions.

(I) There exists $\eta, R>0$ such that for every $\sigma, s \in I, \sigma<s$, $(x, t) \rightarrow$ $F(x, t)$ is a continuous mapping of $B(s, R) \times(-\eta, \eta)$ into $X_{\sigma}$.

(II) There exists a constant $C$ such that for every $t,|t|<\eta$, all $u$ and $v$ with $u, v \in B(s, R)$ and all $\sigma, s \in I$ with $\sigma<s$,

$$
\operatorname{Sup}_{|t| \leq \eta}\|F(u, t)-F(v, t)\|_{\sigma} \leqq \frac{C}{s-\sigma}\|u-v\|_{s} .
$$

(III) $F(0, t) \in C\left[[-\eta, \eta), X_{s}\right]$ and there exists a constant $K$ such that

$$
\operatorname{Sup}_{s \in I} \operatorname{Sup}_{|t| \leqq \eta}\|F(0, t)\|_{s} \leqq K .
$$


Theorem 3.1. (Nishida). Under hypotheses I, II, and III there exists a constant $a<\eta$ and $a$ unique function $x(t)$ such that for every $b, s \in I, s<b$, $x(t) \in C^{1}\left[(-a(b-s), a(b-s)), X_{s}\right], x(t) \in B(s, R)$ and for $|t|<a(b-s)$, $x(t)$ satisfies (3.1).

PROOF. The proof is given in [23] if one makes appropriate changes in the interval $I$.

In [3] Baouendi and Goulaouic give an existence and uniqueness theorem for nonlinear singular problems of the form

$$
\frac{d x(t)}{d t}-\frac{\Lambda x(t)}{t}=F(x(t), t)
$$

where $\Lambda$ is a bounded operator. These results contain Theorem 3.1 as a special case $(\Lambda=0)$.

Remark. Theorem 3.1 is used to derive the most general version of the nonlinear Cauchy-Kowalewski Theorem (see [22]).

We next consider a different nonlinear version of Theorem I.2.1. because of the unusual form of the scale estimate this result uses. This theorem is based on the observation that in the linear case the condition

$$
\|A x\|_{\sigma} \leqq C(s-\sigma)^{-1}\|x\|_{s}
$$

can be replaced by

$$
\|A x\|_{s} \leqq C(\partial / \partial s)\|x\|_{s} .
$$

We need the following conditions.

(IV) There exists a function $G(t)$ for $0 \leqq t<\infty$ such that $G \geqq 0$, $G^{\prime} \geqq 0, G^{\prime \prime} \geqq 0$. Moreover, for all $x, y \in B(s, R)$,

$$
\|F(x, t)-F(y, t)\|_{s} \leqq\left(1+\frac{\partial}{\partial s}\right)\left[G\left(\|x\|_{s}+\|y\|_{s}\right)\|x-y\|_{s}\right] .
$$

(V) $F(0, t) \in B(s, R)$.

We also need an additional restriction on the scale $S$.

(VI) If $x \in S$, then $\|x\|_{s}$ is convex downward and for all $x, y \in S$,

$$
(\partial / \partial s)\|x+y\|_{s} \leqq(\partial / \partial s)\|x\|_{s}+(\partial / \partial s)\|y\|_{s} .
$$

THEOREM 3.2 (Ovcyannikov). Under the hypotheses I, IV, V, and VI there exists $k>0$ and a unique function $x(t)$ such that if $s \in I$ and $\Delta_{s}=$ $\{t ; s+k t<R, t \geqq 0\}$, then $x(t) \in C^{1}\left[\Delta_{s}, X_{s}\right], x(t) \in B(s, R), t \in \Delta_{s}$, and $x(t)$ satisfies (3.1.)

Proof. The proof was given in [25] where this result was used to discuss 
certain nonlinear problems occurring in hydrodynamics. This result can also be obtained from the previous theorem.

It would also be extremely useful to have a nonlinear version of Theorem II.1.1.

4. Lie algebras and scales. In this section we shall point out that the scales we have introduced are related to the notion of an analytic vector for a Lie algebra. Let $X$ be a Banach space with $\|\cdot\|$. Let $A_{i}, 1 \leqq i \leqq N$, be closed linear operators on $X$. We assume that the set $\mathscr{A}=\left\{\sum_{i=1}^{N} \alpha_{i} A_{i}\right.$, $\left.\alpha_{i} \in \mathbf{C}\right\}$ is a finite dimensional Lie algebra with

$$
\left[A_{i}, A_{j}\right]=A_{i} A_{j}-A_{j} A_{i}=\sum_{k=1}^{N} \beta_{i j k} A_{k}
$$

for some $\beta_{i j k} \in \mathbf{C}$ and the $A_{i}$ are linearly independent. In this circumstance we will define

$$
\begin{gathered}
\left.\|x\|_{s}=\|x\|_{d, p s}=\left(\sum_{n}\left\|A^{n} x\right\| s^{|n|} / n !^{d}\right)^{p}\right)^{1 / p}, \\
X_{s}=X_{d, p, s}=\left\{x \in X,\|x\|_{s}<\infty\right\}
\end{gathered}
$$

where $0<s<\infty, 1 \leqq p \leqq \infty, 0 \leqq d<\infty, n=\left(n_{1}, n_{2}, \ldots, n_{N}\right), n_{i} \geqq 0$,

$$
A^{n}=A_{1}^{n_{1}} A_{2}^{n_{2}} \cdots A_{N}^{n_{N}},
$$

$|n|=n_{1}+n_{2}+\cdots+n_{N}, d=\left(d_{1}, d_{2}, \ldots, d_{n}\right), d_{i} \geqq 0$, and

$$
n !^{d}=n_{1} !^{d_{1}} \cdots n_{N} !^{d_{N}},
$$

( $p=\infty$ gives the sup norm).

Proposition 4.1. $S=\left\{X_{s},(0, \infty)\right\}$ is a scale.

Proof. This is easy.

Remark. In general one would expect $X_{s}$ to consist of the zero element. We will now explore when this is not the case.

In many of the applications the Lie algebra consists of one differential operator $A$ in which case $n$ is an integer and $d$ is a real number. The following result shows that the scales generated are nontrivial.

Theorem 4.1 (Beals). Let $\mathscr{A}=\{c A, c \in \mathbf{C}\}$. Suppose that the spectrum of $A$ is contained in a region of the form

$$
\operatorname{Re}(q)<a+b|q|^{\beta}
$$

for some constants $a, b, \beta, 0 \leqq \beta<1$. Suppose that for q outside this region, the resolvent operator satisfies

$$
\left\|(q I-A)^{-1}\right\| \leqq c(1+|q|)^{N}
$$


for some constants $c, N$. Then $\bigcup_{s>0} X_{d, p, s}$ is dense in $X$ for any $p, 1 \leqq p \leqq \infty$, and $d>1$.

PROOF. The proof is an elementary modification of the proof of Theorem $1.1,[5]$.

At this point it is not clear that Lie algebras play any role. The next example illustrates that, in fact, Lie algebras do play a role. This example has interesting applications in quantum mechanics [28].

Proposition 4.2. Let $A$ and $B$ be closed operators on a Banach space $X$ with norm $\|\cdot\|$. Let $\alpha, \beta$ satisfy $0<\alpha<1,0<\beta<\mathrm{x}$, and suppose

$$
\|x\|_{s}=\|x\|_{\alpha, \beta, p, s}=\left(\sum\left(\left\|B^{m} A^{n} x\right\| s^{n+m}(m !)^{-\alpha}(n !)^{-\beta}\right)^{p}\right)^{1 / p}
$$

and $X_{s}=X_{\alpha, \beta, p, s}=\left\{x ;\|x\|_{s}<\infty\right\}$. If $[A, B]=I$, then

$$
\|A x\|_{\sigma} \leqq C(s-\sigma)^{-\beta}\|x\|_{s}, \sigma<s,
$$

and

$$
\|B x\|_{\sigma} \leqq C\left((s-\sigma)^{-\alpha}+(s-\sigma)^{\beta-1}\right)\|x\|_{s}, \sigma<s .
$$

Proof. We compute

$$
\|A x\|_{\sigma} \leqq(\sigma)^{-1} \sup \left((n+1)^{\beta}(\sigma / s)^{n+1}\right)\|x\|_{s} \leqq C(s-\sigma)^{-\beta}\|x\|_{s} .
$$

By induction we can show that $A^{n} B=B A^{n}+n A^{n-1}$, and consequently

$$
\begin{aligned}
\|B x\|_{\sigma} \leqq & \left(\sum\left(\left\|B^{m+1} A^{n} x\right\|(\sigma)^{n+m}(m !)^{-\alpha}(n !)^{-\beta}\right)^{p}\right)^{1 / p} \\
& +\left(\sum\left(n\left\|B^{m} A^{n-1} x\right\|(\sigma)^{n+m}(m !)^{-\alpha}(n !)^{-\beta}\right)^{p}\right)^{1 / p} \\
\leqq & (\sigma)^{-1} \sup \left((m+1)^{\alpha}(\sigma / s)^{m+1}\right)\|x\|_{s} \\
& +s \sup n^{(1-\beta)}(\sigma / s)^{n}\|x\|_{s} \\
\leqq & C\left((s-\sigma)^{-\alpha}+(s-\sigma)^{\beta-1}\right)\|x\|_{s} .
\end{aligned}
$$

We are again left with the problem of the non-triviality of the spaces $X_{\alpha, \beta, p, s}$ which is answered by the following theorem.

THEOREM 4.2. Let $X=L^{2}$ be the space of square integrable functions on the real line with the usual norm and for $f \in X, A f=d f / d x, B f=x f$. Let $X_{\alpha, \beta, 2, s}$ be defined as in Proposition 4.2. If $\alpha>0, \beta>0, \alpha+\beta>1$, then $X_{\alpha, \beta, 2, s}$ is dense in $X$. If $\alpha>0, \alpha+\beta=1$ and $s$ is sufficiently small, then $X_{\alpha, \beta, 2, s}$ is dense in $X$. If $\alpha>0, \beta>0, \alpha+\beta<1$, then $X_{\alpha, \beta, 2, s}=\{0\}$.

Proof. This follows from Lemma 5.1 in [14] and a minor extension of the proof of Corollary 5.1 in [14]. Here we obtain the density of $X_{\alpha, \beta, 2, s}$ in $X$ because $X$ is a (weighted) $L^{p}$-space. As $X_{\alpha, \beta, 2, s}$ is not a weighted $L^{p}$-space, it seems impossible to generalize this to prove that $X_{\alpha, \beta, 2, s}$ is dense in $X_{\alpha, \beta, 2, \sigma}$ for $0<\sigma<s$ (see page 236 of [12 v. 2]). 
EXAMPLE. In the above we can choose $\alpha=\beta=1 / 2$ and then we have $X_{s}=X_{1 / 2,1 / 2,2, s}$ is dense in $X$ for $s$ small. Also

$$
\begin{aligned}
\|A x\|_{\sigma} & \leqq C\left(s-s^{\prime}\right)^{-1 / 2}\|x\|_{s}, \\
\|B x\|_{\sigma} & \leqq C\left(s-s^{\prime}\right)^{-1 / 2}\|x\|_{s},
\end{aligned}
$$

and, consequently, and quadratic expression in $A$ and $B$ is of type 1 on $X_{s}$. We can now apply our theory to examples such as the prarmetric oscillator equation, see Section 10.

Remark. In the above we have not shown that $X_{s}$ is dense in $X_{\sigma}$ for $\sigma<s$. What we have shown is that $X_{s}$ is a fairly large space. We note that much of the previous theory does not depend on the density of $X_{s}$ in $X_{\sigma}$; in fact, just the duality results depend on this density. However, the duality results have many important applications in P.D.E.'s and thus we believe that the density of $X_{s}$ in $X_{\sigma}$ is a most interesting question.

Because of the complexity of the description of the scales of spaces used in these notes, it is difficult to believe that they could be intrinsic. However, if we define $Y_{d, p}=U_{s>0} X_{d, p, s}$, then the techniques used in [38] will show that $Y_{d, p}$ is independent of $p$. We claim that if an initial value problem is "well posed" in the scale $X_{d, p, s}$, then the number $d$ measures how far the problem is from being classically well posed. Consequently the number $d$ is intrinsic to the problem. Clearly the numbers $p$ and $s$ are not intrinsic. for more details on this point, see [28].

\section{REFERENCES}

1. M. S. Baouendi and C. Goulaouic, Cauchy problems with characteristic initial hypersurface, Comm. Pure Appl. Math., 26 (1973), 455-475.

2. - - Singular nonlinear Cauchy problems, J. Diff. Equ. 22 (1976), 268-291.

3. - Remarks on the abstract form of nonlinear Cauchy-Kovalevsky theorems, Comm. Partial Diff. Equ. 2 (11) (1977), 1151-1162.

4. - Pseudodifferential nonlinear Cauchy problems and applications, To appear.

5. R. Beals, Hyperbolic equations and systems with multiple characteristics, Arch. Rat. Mech. Ana., 48 (1972), 123-152.

6. Y. Bruhat, Diagonalisation des systèmes quailinéaries et hyperbolicité non stricte, J. Math., 45 (1966), 371-386.

7. P. DuChateau, A Holmgren type theorem for pseudo-differential operators in Gevrey classes, J. Diff. Eqns., (1973), 319-328.

8. - New proofs and generalizations of theorems of existence and uniqueness for the Goursat problem, Applicable Analysis, 2 (1972), 61-78.

9. - The Cauchy-Goursat problem, Memoirs A.M.S., 118 (1972).

10. P. DuChateau and J. F. Treves, An Abstract Caucy-Kowalewski theorem in scales of Gevrey Classes, Symposia Math. 7, Academic Press, New York, 1971.

11. A. Friedman, A new proof and generalizations of the Cauchy-Kowalewski theorem, Trans. A.M.S., 98 (1961), 1-20. 
12. I. M. Gel'fand and G. E. Shilov, Generalized Functions, Academic Press, New York, Vol. 2, 1968; Vol. 3, 1967.

13. Gevrey, Sur la nature analytique des solutions des equations aux dirivees partielles, Ann. de l'Ecole Norm. Sup. 35 (1917), 129-189.

14. R. Goodman, Differential operators of infinite order on a Lie Group I, J. Math. Mech., 19 (1970), 879-894.

15. L. Hormander, Linear Partial Differential Operators, Springer, Berlin, 1963.

16. A. Lax, On Cauchy's problem for partial differential equations with multiple characteristics, Comm. Pure Appl., 9 (1956), 135-169.

17. J. Leray, Equations hyperboliques non-strictes: contre-examples, du type DeGeorgi, ano théorèmes d'existence et d'unicite, Math. Annalen 162 (1966), 228-236.

18. J. Leray and Y. Ohya, Equations et systems non linéaires, hyperboligues non stricts, Math. Annalen, 170 (1967), 167-205.

19. J. L. Lions and E. Magenes, Non-Homogeneous Boundary Value Problems and Applications, III, Springer-Verlag, New York, 1973.

20. M. Nagumo, Über das Anfangswertproblem Partieller Differential gleichungen, Japan J. Math., 18 (1941), 41-47.

21. E. Nelson, Analytic vectors, Annals. Math., 70 (1959), 572-615.

22. L. Nirenberg, An abstract form of the nonlinear Cauchy Kowalewski theorem, J. Diff. Geom., 6 (1972), 561-576.

23. T. Nishida, $A$ note on the Nirenberg's theorem as an abstract form of the nonlinear Cauchy-Kowalewski theorem in a scale of Banach spaces, To appear in J. Diff. Geometry.

24. Y. Ohya, Le probleme de Cauchy pour les equations hyperboliques a characteristique mutiple, J. Math. Soc. Japan, 16 (1964), 268-286.

25. L. V. Ovcyannikov, A nonlinear Cauchy problem in a scale of Banach spaces. Dokl. Akad. Nauk SSR 200 (1971), 789-792; Soviet Math. Dokl. 12 (1971), 1497-1502.

26. - - Singular operators in Banach scales, Dokl. Akad. Nauk. SSR 163, 819-822; Soviety Math. Dokl. 6(1965), 1025-1028.

27. J. Persson, Global Goursat problems for functions of Gevrey-Lednev type, Ann. Sci. Norm. Sup. Pisa, 23 (1969), 387-412.

28. - Linear characteristic Cauchy problems for partial differential equations with variable not only time dependent coefficients, Bullettino U.M.I., 44 (1971), 91-102.

29. - Linear Goursat problems for entire functions when the coefficients are variable, Ann. Sci. Norm. Sup. Pisa, 23 (1969), 87-98.

30. - On the local and global non-characteristic Cauchy problem when the solutions are holomorphic functions of analytic functionals in the space variables, Arkiv for Mat. 9 (1971), 171-180.

31. - On the supports of solutions of linear partial differential equations with analytic coefficients, Annali di Mat. Pura Appl. (IV) 91 (1972), 79-96.

32. - On uniqueness cones, velocity cones and P-convexity. Annali di Mat. Pura Appl., to appear.

33. S. Steinberg, Applications of linear programming theory to existence and uniqueness classes for the Cauchy problem, Annali di Mat. Pura Appal. (IV) 64 (1977), 69-85.

34. - Existence and uniqueness of solutions of hyperbolic equations which are not necessarily strictly hyperbolic, J. Diff. Eqn., 17 (1975), 119-153.

35. - Infinite systems of ordinary differential equations with unbounded coefficients and moment problems, J. Math. Anal. Appl. 41 (1973), 685-694.

36. - Local groups and analytic vectors, Technical Report, Purdue University, 1971.

37. - The Cauchy problem for differential equations of infinite order, J. Diff. Eqn. 9 (1971), 591-607. 
38. S. Steinberg and F. Treves, Pseudo-Fokker Planck equations and hyperdifferential operators, J. Diff. Eq. 8 (1970), 333-366.

39. G. Talenti, Osservazioni sulla nota: Un problema d. Cauchy, Ann. Sc. Norm. Sup. Pisa, 19 (1965), 179-184.

40. —, Un problema di Cauchy, Ann. Scoula Norm. Sup. Pisa Cl. Sc., 3, 18(1964), $165-186$.

41. F. Treves, An abstract nonlinear Cauchy-Kowalewski theorem, Trans. Amer. Math. Soc., 150 (1970), 77-92.

42. - On the theory of linear partial differential operators with analytic coefficients, Trans. Amer. Math. Soc., 137 (1969), 1-20.

43. - Ovcyannikov theorem and hyperdifferential operators, Rio de Janeiro, I.M.P.A., 1969.

44. - Topological Vector Spaces, Distributions and Kernels, Academic Press, New York, 1967.

45. H. Yamagata, The nonlinear abstract Cauchy-Kowalewski theorem described in the form of ranked spaces, Proc. Japan Acad. 49 (1973), 601604.

46. T. Yamanaka, Note on Kowalewski's system of partial differential equations, Comment. Math. Univ. St. Paul 9 (1960), 7-10.

47. T. Yamanaka and J.Persson, On an extension of Holmgren's uniqueness theorem, Comment. Math. Univ. St. Paul, XXII-1 (1973), 19-30.

The University of New MeXico, Albuquerque, NM 87131. 